populations. In the current study, we aimed to assess the prevalence of hyperuricemia in a Chinese population.

Method: We recruited a total of 4221 participants from a crosssectional study, comprising 1892 type 2 diabetes patients and 2329 subjects with normal glucose tolerance (NGT). All cases were unrelated type 2 diabetes patients recruited from the inpatient database of Shanghai Diabetes Institute. The NGT subjects were assessed by standard $75 \mathrm{~g}$ OGTTs, and with negative family history of diabetes that recruited from Shanghai Diabetes Study and Shanghai Diabetes Study II. Hyperuricemia was defined as the concentration of serum uric acid $>420 \mathrm{mmol} / \mathrm{l}$ in males and serum uric acid $>357 \mathrm{mmol} / \mathrm{l}$ in females. In our study, most patients were not on a regular treatment for hyperuricemia. Phenotypes for anthropometric and biochemical traits related to uric acid were extensively measured for all participants.

Result: The overall prevalence of hyperuricemia was $11.16 \%$ in NGT subjects and $17.02 \%$ in type 2 diabetes patients. In females, the prevalence of hyperuricemia was significant higher in type 2 diabetes patients than in those with NGT $(20.39 \%$ vs. $7.63 \%, p<0.001)$. In males, it was opposite that the prevalence of hyperuricemia showed a tendency to be higher in NGT subjects than in type 2 diabetes patients $(17.05 \%$ vs. $13.88 \%, p=0.0588)$. Then we assessed the prevalence of hyperuricemia stratified by age in both NGT subjects and type 2 diabetes patients. The prevalence of hyperuricemia increased with increasing age in females in both NGT subjects and type 2 diabetes patients $(p<0.0001$ in NGT subjects and $p<0.0001$ in type 2 diabetes patients). However, in males, the results were not significant $(P=0.4294$ in NGT subjects and $p=0.3966$ in type 2 diabetes). We also compared the prevalence of hyperuricemia between NGT subjects and the type 2 diabetes patients in females and males. The results showed that when age $<55$ in females, the prevalence of hyperuricemia was significant higher in type 2 diabetes patients than in those with NGT $(p<0.0001$ when age $<45$ and $p=0.0037$ when $45 \leqslant$ age $<55$ ). While in males, there is a obvious lower prevalence in type 2 diabetes patients than in those with NGT $(p<0.0001$ when $45 \leqslant$ age $<55$ and $p=0.0037$ when $55 \leqslant$ age $<65)$.

Conclusion: Hyperuricemia is prevalent in Chinese type 2 diabetes patients and the age-associated prevalence of hyperuricemia was significant high in females. Therefore, strategies to prevent and treat hyperuricemia in type 2 diabetes patients are urgently needed in order to stem this national pandemic.

\section{PO360 \\ IS PROSTATIC SERUM ANTIGEN CORRELATED WITH INSULIN RESISTANCE IN MEN WITH TYPE 2 DIABETES MELLITUS-METS}

$\underline{\text { H. Susanto }}{ }^{1,1}$, A. Tjokroprawiro ${ }^{1}$, S. Wibisono ${ }^{1}$, S. Murtiwi ${ }^{1}$, S. Adi ${ }^{1}$, A. Sutjahjo ${ }^{1}$, A. Pranoto ${ }^{1}$. ${ }^{1}$ Surabaya Diabetes and Nutrition Center dr. Soetomo Teaching Hospital Airlangga University Faculty of Medicine, Surabaya, Indonesia

Background: Metabolic syndrome (MetS) is a cluster of cardiovascular risk factors that includes hypertension, diabetes mellitus, obesity, hypertriglyceridemia, and low high-density lipoprotein cholesterol, with insulin resistance as the underlying hallmark feature. The prevalence of MetS has been increasing worldwide and has become a major public health problem in many western countries.

Recently, increasing evidences suggests that MetS may be involved in the development and progression of certain types of cancer as an independent etiologic factor including breast cancer, endometrial cancer, colorectal cancer, pancreatic cancer and prostate cancer. MetS was firstly observed as a composite factor associated with prostate cancer risk in 2004, and more studies have since reported the association between MetS and prostate cancer.
In biologic models proposed to explain this association, researchers note the higher concentrations of insulin and insulin-like growth factor 1 (IGF-1) in early diabetes and the lower testosterone and IGF-1 levels and higher estrogen concentrations in long-term diabetes, Whether diabetes influences levels of biomarkers such as Prostate-Specific Antigen (PSA), which is involved in the detection of prostate cancer, is still unknown.

Factors influencing serum PSA levels in men include age, benign prostatic hyperplasia, prostatitis, and Body Mass Index (BMI). In this study, we examined whether PSA on the person who have insulin resistance which is often happen in men with Type 2 Diabetes Mellitus- Metabolic Syndrome.

Method: This cross sectional study was performed in Surabaya private clinic. Study period was six months between January to June 2014. Plasma concentrations of glycated haemoglobin (HbA1c) and fasting and post-prandial glucose were analysed and homeostatic model assessment of insulin resistance (HOMA-IR) were calculated. HOMA-IR value more than 2.0 is considered to be positive for IR. Statistical analysis was performed using SPSS 17.0 and Spearmen's test.

Result: Fifty two male subjects were enrolled in this study. The mean age was $62.32 \pm 8.72$ years old, fasting blood sugar $139.68 \pm 43.65 \mathrm{mg} / \mathrm{dL}$, mean A1C $7.89 \pm 1.80 \%$, the mean HOMA-IR 7.14 \pm 4.77 , and the mean of PSA value was $3.76 \pm 1.99 \mathrm{ng} / \mathrm{dL}$. The PSA showed negative correlation with Insulin Resistance $(r=-0.049 ; \mathrm{p}<0.005)$.

Conclusion: The level of PSA had negative correlation with insulin resistance among men with T2DM-MetS in this study.

\section{PO361}

TESTOSTERON LEVEL AND INSULIN RESISTANCE IN MEN WITH TYPE 2 DIABETES MELLITUS-METABOLIC SYNDROME

A. Tjokroprawiro ${ }^{1}, \underline{\text { H. Susanto }}{ }^{1}$, S. Wibisono ${ }^{1}$, S. Murtiwi $^{1}$, S. Adi ${ }^{1}$, A. Sutjahjo ${ }^{1}$, A. Pranoto ${ }^{1} .{ }^{1}$ Surabaya Diabetes and Nutrition Center dr. Soetomo Teaching Hospital Airlangga University Faculty of Medicine, Surabaya, Indonesia

Background: Testosterone deficiency often occurs in people with type 2 diabetes mellitus (T2DM) where it contributes to mood and libido disturbances. Total testosterone levels inversely associated with insulin resistance, a factor which has the potential to provide micro and macro vascular complications in patients with T2DM.

Several studies in men showed that testosterone supplementation can improve insulin sensitivity conditions. Recent studies have reported that $20-64 \%$ of men with testosterone deficiency/hypogonadism also have T2DM. The proportion of men who have both T2DM and hypogonadism increases in older groups of men.

Men who have slightly reduced total testosterone level, but not low enough to be considered as testosterone deficiency are also more likely to have low insulin and high blood glucose levels. This study will examine the correlation between total testosterone level and insulin resistance in men with T2DMMetS.

Method: This cross sectional study was performed in Surabaya private clinic. Study period was six months between January to June 2014. The variables include: fasting blood sugar, 1 hour post prandial, A1C, HOMA-IR and levels of the total testosterone was measured by radioimmunoassay technique. Low testosterone for patient with T2DM was defined as below $400 \mathrm{ng} / \mathrm{dL}$ and normal if above $400 \mathrm{ng} / \mathrm{dL}$. HOMA-IR value more than 2.0 is considered to be positive for IR.

Patients on testosterone replacement, and corticosteroids were excluded from this study. Statistical analysis was performed using SPSS 17.0 and Spearmen's test.

Result: Fifty two male subjects were enrolled in this study. The mean age was $62.32 \pm 8.72$ years old, fasting blood sugar $139.68 \pm 43.65 \mathrm{mg} / \mathrm{dL}$, blood glucose 1 hour post prandial $255.56 \pm 112.06 \mathrm{mg} / \mathrm{dL}$, mean A1C $7.89 \pm 1.80 \%$, the 
mean HOMA-IR 7.14 \pm 4.77 , while the mean testosterone levels $315.39 \pm 251.30 \mathrm{ng} / \mathrm{dL}$.

The statistical analysis showed inverse correlation between total testosterone levels and insulin resistance $(r:-0.215$, p: 0.034)

Conclusion: There was a significant inverse correlation between total testosterone level and insulin resistance in this study.

\section{Reference(s)}

Bonora E., Kiechl S., Willeit J. 1998. Prevalence of Insulin Resistance in Metabolic Disorders: The Bruneck Study. Diabetes 47: 1643-16.

Chiang J., Lai N., Chang J. 2011. Predicting insulin resistance using the triglyceride-to-high-density lipoprotein cholesterol ratio in Taiwanese adults. Cardiovascular Diabetology. 10: 93.

Gonzalez-Chavez A., Simental-Mendia L., Elizondo-Argueta S. 2011. Elevated triglycerides/HDL-cholesterol ratio associated with insulin resistance. Cir Cir 79: 115-119.

\section{PO362}

A HYDROPHILIC DERIVATIVE OF PROBUCOL AMELIORATES GLUCOSE TOLERANCE AND INSULIN SENSITIVITY IN HFD-FED MICE

$\underline{\text { K. Takenokuma }}{ }^{1}$, L. Miyamoto ${ }^{1}$, Y. Tomida ${ }^{1}$, Y. Tsuchihashi $^{1}$, T. Matsushita ${ }^{2}$, H. Hattori ${ }^{2}$, H. Nemoto ${ }^{2}$, K. Tsuchiya ${ }^{1}$. ${ }^{1}$ Medical Pharmacology, Inst. Health Biosciences, The University of Tokushima Graduate School, 770-8505, ${ }^{2}$ Pharmaceutical Chemistry, Inst. Health Biosciences, The University of Tokushima Graduate School, 770-8505, Tokushima, Japan

Background: Probucol is an anti-hyperlipidemic agent possessing remarkable antioxidant activity clinically used in some countries including Japan. Although probucol is highly hydrophobic, its succinic acid ester, succinobucol, was reported as a water-soluble derivative and was in clinical trials. In animal models, compared to probucol, succinobucol was shown to be potent in lipid-lowering, anti-inflammatory and anti-atherogenic effects. Furthermore, succinobucol lowered $\mathrm{HbA1c}$ in diabetic patients. The clinical trials finally resulted in failure, but modulating watersolubility of established active compounds seems to be a promising strategy for drug discovery. Meanwhile, we have developed a novel strategy to endow hydrophobic agents with much hydrophilicity using branched oligoglycerols as an appropriate approach to intensify water solubility. Therefore we synthesized a novel highly hydrophilic derivative of probucol, Probucol-(glutaric branched-triglycerol)2 (ProBGL ${ }_{2}$ ) and evaluated its effects on glucose and lipid metabolism in HFD-fed mice.

Method: We orally administrated $1 \mathrm{~g} / \mathrm{kg} /$ day of probucol or $\mathrm{ProBGL}_{2}$ for a week to male ddY mice fed HFD (24 wk), and then performed ipGTT. We also determined plasma insulin and lipid parameters.

Result: Although body weight and food intake were not affected, glucose tolerance was much ameliorated in probucol and ProBGL 2 groups. In the both groups, fasting plasma insulin levels and HOMA-IR were 70\% decreased, suggesting increase in insulin sensitivity. However, even though probucol lowered plasma total cholesterol concentration more than $50 \%$, ProBGL 2 did not affect it. Moreover, TBARS assay showed $\mathrm{ProBGL}_{2}$ did not possess antioxidant activities against lipids in contrast to probucol. Plasma TG and NEFA levels were not altered.

Conclusion: Our data show that probucol and its hydrophilic derivative, ProBGL $\mathrm{B}_{2}$ improve glucose intolerance and insulin resistance in HFD-fed mice and those are unrelated to the canonical potency of probucol such as lipid-lowering or antioxidant activities. ProBGL ${ }_{2}$ should be a novel potent antidiabetic agent.
PO363

UNRAVELLING PATHWAYS CONTRIBUTING TO THE ASSOCIATION BETWEEN BIRTHWEIGHT AND PREDIABETES: AN ANALYSIS USING STRUCTURAL EQUATION MODELING

C.H.T.H. Tam ${ }^{1}$, X.L. Yang ${ }^{1}$, A.O.Y. Luk ${ }^{1}$, P.C.Y. Tong ${ }^{1}$, A.P.S. Kong ${ }^{1}$, W.Y. So ${ }^{1}$, W.H. Tam ${ }^{2}$, J.C.N. Chan ${ }^{1}$, R.C.W. Ma ${ }^{1}$ ${ }^{1}$ Department of Medicine and Therapeutic, ${ }^{2}$ The Chinese University of Hong Kong, Hong Kong, Hong Kong

Background: Recent studies demonstrated a U-shaped relationship between birthweight and future risk of T2D, leading to hypotheses of long-term programming effects of genetic factor and intrauterine environment on later risk of diabetes in the offspring. We aim to understand the pathways contributing to the association between birthweight and prediabetes by using three independent cohorts from different stages of the lifecourse (children, adolescents and adults) in the Chinese population.

Method: Parental history of diabetes, birthweight and clinical data including anthropometric traits and fasting plasma glucose were collected from 965 children (mean age 7.0 \pm 0.4 years, $52.0 \%$ male), 2,026 adolescents (mean age 15.6 \pm 2.0 years, $45.5 \%$ male) and 555 adults (mean age $43.5 \pm 8.2$ years, $48.3 \%$ male). Association between participants' birthweight and parental diabetes status was tested by multiple linear regression analysis, with participants' sex and duration of gestation as covariates. We used structural equation modeling (SEM) to examine the interrelationship between parental diabetes, birthweight, FPG and/or obesity traits, and estimate the mediation effects of birthweight and obesity in the association between parental diabetes and FPG level.

Result: We observed that maternal diabetes was significantly associated with higher birthweight $\left(P=2.2 \times 10^{-7}\right)$ while paternal diabetes was nominally associated with lower birthweight $(P=0.089)$, compared with individuals without parental diabetes. Using the SEM approach, we provide strong evidence that maternal diabetes was directly associated with increased future risk of obesity $(P=0.006)$ and hyperglycaemia $(P=0.037)$, as well as weaker evidence that paternal diabetes was associated with increased later hyperglycaemia risk $(P=0.071)$. These associations were also partially mediated by birthweight and/or obesity, with higher birthweight and/or risk of obesity observed in individuals with maternal diabetes, whereas lower birthweight was observed in individuals with paternal diabetes $\left(P_{\text {mediation }}<0.05\right)$.

Conclusion: We have shown that the association between parental diabetes and offspring prediabetes were partially mediated by birthweight and/or obesity. Our findings support the notion that both genetic factor and intrauterine environment contribute to the association between birthweight and future risk of hyperglycaemia. Obesity is a key mediator in the pathogenesis of T2D, particularly in individuals with parental diabetes. This has important implications regarding targeting individuals to break the transgenerational effects of maternal diabetes.

Acknowledgement: RCWM acknowledge funding support from the Research Grants Council General Research Fund (CU471713). 